\title{
Nascimento da Raia-viola, Zapteryx brevirostris (Müller \& Henle) (Chondrichthyes, Rhinobatidae), em cativeiro
}

\author{
Manoel M. B. Gonzalez \\ Núcleo de Pesquisa e Estudo em Chondrichthyes (NUPEC). Rua Ana Pimentel 12, 11030-050 Santos, São Paulo, Brasil. E- \\ mail:gonzalez@nupec.com.br
}

\begin{abstract}
Birth of guitarfish, Zapteryx brevirostris (Müller \& Henle) (Chondrichthyes, Rhinobatidae) in captivity. A female of Zapteryx brevirostris were caught at Queimada Grande Island, Itanhaém, São Paulo on 19/II/ 2002, and transported to Núcleo de Pesquisa e Estudo em Chondrichthyes (NUPEC) captivities. At 21/II/2002, the female gave birth to six pups (three males and three females). This is a first birth record of $Z$. brevirostris in captivity.

KEY WORDS. Captive, reproduction.
\end{abstract}

RESUMO. Um exemplar fêmea de Zapteryx brevirostris foi capturada na Ilha Queimada Grande, Itanhaém, São Paulo em 19/II/2002, e transportada para o cativeiro do Núcleo de Pesquisa e Estudo em Chondrichthyes (NUPEC). Em 21/II/2002, a fêmea pariu seis filhotes (três machos e três fêmeas). Este é o primeiro registro de nascimento de Z. brevirostris em cativeiro.

PALAVRAS-CHAVE. Cativeiro, reprodução.

O número de elasmobrânquios capturados e mantidos em cativeiro vem aumentando durante os últimos oito anos (GoNZALEZ 1996-2002). Um dos motivos prováveis para este aumento estaria na popularidade do grupo e seus caracteres específicos relacionados ao comportamento, fisiologia, anatomia e reprodução (Demski 1990). Conseqüentemente um número maior de espécies se reproduzem com sucesso em cativeiro como exemplares das famílias Carcharhinidae e Myliobatidae (UCHIDA et al. 1990).

De acordo com o International Zoo Yearbook (1963-2003), 47 espécies de 13 famílias de tubarões e 31 espécies de nove famílias de raias nasceram em cativeiro. As espécies assim obtidas eram provenientes de aquários, parques marinhos e demais instituições que trabalham com elasmobrânquios cativos, possuindo apenas três registros para indivíduos da família Rhinobatidae: Rhinobatos lentiginosus (Garman, 1880), Rhinobatos productus (Girard, 1854) e Rhinobatos hynnicephalus (Richardson 1846).

A raia-viola (Fig. 1) ocorre do Nordeste do Brasil ao Norte da Argentina (Figueiredo 1977, Menni \& Stehmann, 2000), alcançando comprimento máximo de $100 \mathrm{~cm}$, possuindo viviparidade aplacentária como modo reprodutivo (BATISTA 1987, Намletт \& Коов 1999). Pode ser capturada em águas costeiras, com até $120 \mathrm{~m}$ de profundidade (Figueiredo 1977). Objetivou-se neste trabalho demonstrar pela primeira vez, o nascimento e a manutenção dos exemplares de $Z$. brevirostris em cativeiro.

\section{MATERIAL E MÉTODOS}

O Núcleo de Pesquisa e Estudo em Chondrichthyes (NUPEC) recebe regularmente animais vivos proveniente da pesca comercial e artesanal do litoral de Santos, Estado de São Paulo, que opera no Sul e Sudeste do Brasil. No dia 19/II/2002, recebemos uma fêmea de raia-viola com aproximadamente 50 cm de comprimento total $\left(\mathrm{T}_{\mathrm{L}}\right)$. O exemplar foi capturado por barco camaroneiro entre 50-70 $\mathrm{m}$ de profundidade próximo à Ilha Queimada Grande $\left(24^{\circ} 29^{\prime} \mathrm{S}, 46^{\circ} 30^{\prime} \mathrm{W}\right)$, distante $35 \mathrm{~km}$ da costa continental do Município de Itanhaém, São Paulo. Após diagnóstico, observamos o abdômen distendido e as nadadeiras pélvicas e região da cloaca edemaciada.

O indivíduo foi acondicionado em quarentena de formato circular com 2500 1, montada sob Sistema Jauber, filtros biológicos (bactérias nitrificantes) e mecânicos (lã de vidro), termômetro digital, iluminação de vapor metálico e sala equipada com aparelho de ar condicionado para manter a temperatura da sala e dos aquários estáveis. Como substrato utilizouse halimeda, com aproximadamente $10 \mathrm{~cm}$ de espessura. Os parâmetros físico-químicos dos sistemas de cativeiros e da quarentena foram monitorados diariamente (Tab. I).

\section{RESULTADOS}

No dia 21/II/2002, houve o nascimento de seis exemplares, sendo três machos (respectivamente, 13,0; 12,5 e 11,5 mm $\mathrm{T}_{\mathrm{L}}$ ) e três fêmeas (respectivamente, 13,0; 13,0 e 12,5 $\mathrm{mm} \mathrm{T}_{\mathrm{L}}$ ). $\mathrm{O}$

Revista Brasileira de Zoologia 21 (4): 785-788, dezembro 2004 

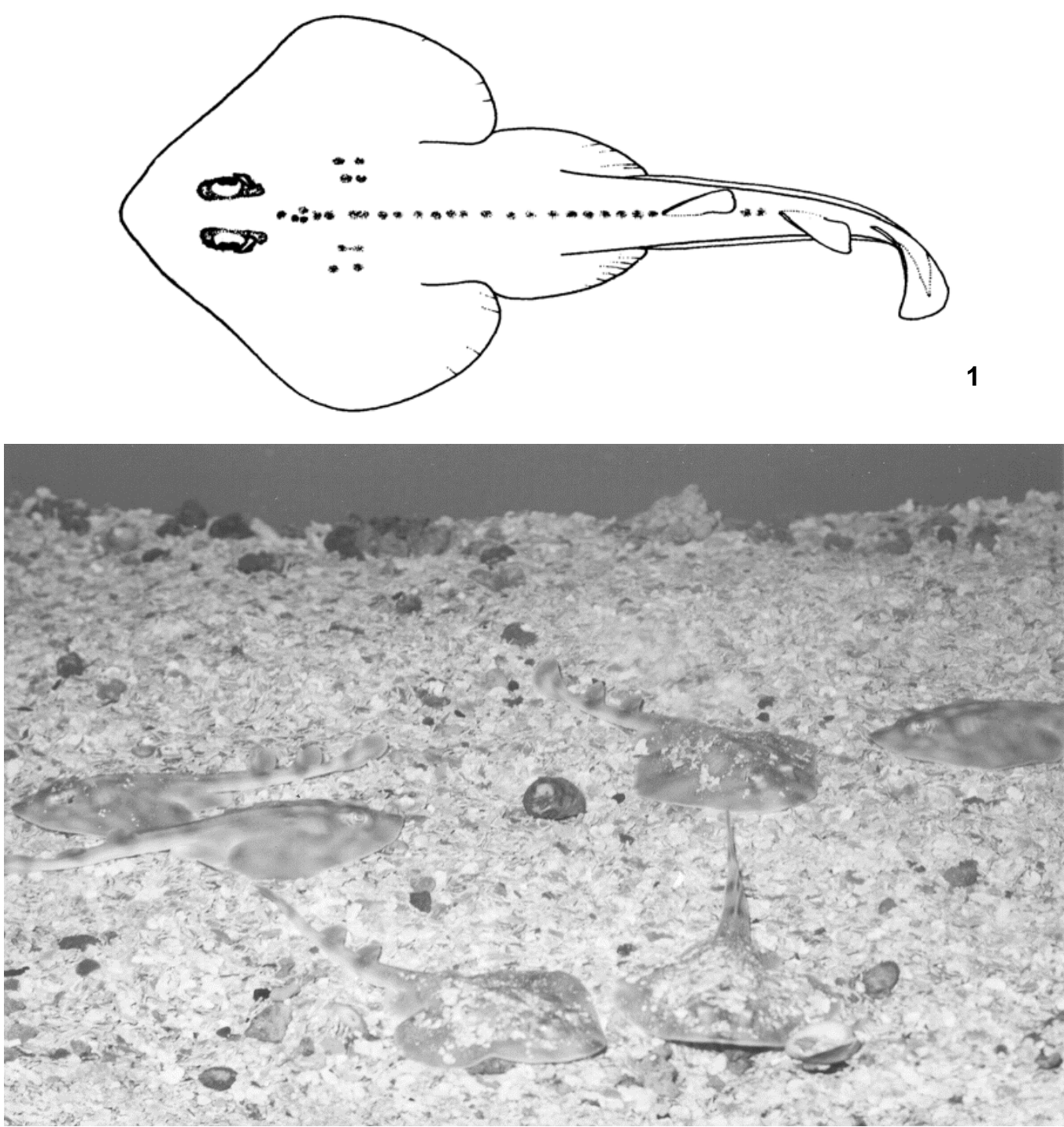

Figuras 1-2. Zapteryx brevirostris : (1) vista dorsal, modificado de FIGUEIREDO (1977); (2) exemplares nascidos em cativeiro. Foto: Aurélio Douglas.

Tabela I. Principais parâmetros físico-químicos médios analisados nos cativeiros do NUPEC. Todos os cativeiros apresentaram valores nulos para nitrito $(\mathrm{mg} / \mathrm{l})$ e amônia $(\mathrm{mg} / \mathrm{l})$.

\begin{tabular}{lccc}
\hline \multicolumn{1}{c}{ Cativeiro } & Salinidade $(\mathrm{ppm})$ & $\mathrm{pH}$ & Temperatura (Co) \\
\hline Sistema 1 & 33,20 & 8,2 & 24,3 \\
Sistema 2 & 33,43 & 8,2 & 22,1 \\
Quarentena & 33,74 & 8,2 & 22,0 \\
Tanque principal & 33,50 & 8,2 & 22,0 \\
\hline
\end{tabular}

nascimento ocorreu durante o período noturno, não sendo possível o registro. Os filhotes foram transferidos para outro tanque (Sistema 2) de 500 I, onde permaneceram isolados. A fêmea foi mantida na quarentena para observação e cuidados (Gruber \& KeYes 1981, MurRu 1990, SMiTH 1992).

Durante o período de iluminação do cativeiro (10 horas/ dia), as raias encontravam-se sob o substrato, estando visíveis apenas os espiráculos. O período de ausência de iluminação era caracterizado por picos degrande movimentação, al ternados por natação sobre o substrato e meia-água. Os filhotes iniciaram a alimentação 14 dias após seu nascimento, sendo ministrados pequenas porções de invertebrados moídos (camarão e lula).

Após a retirada dos filhotes foi realizado o procedimento profilático ministrando à fêmea antibiótico (terramicina -

Revista Brasileira de Zoologia 21(4): 785-788, dezembro 2004 
25 mg/l) por imersão. Após dois dias a raia iniciou sua al imentação com porções de peixes e invertebrados marinhos (camarão e lula). Como observado nos filhotes, o período de maior atividade foi o noturno. No dia 25/III/2002 o exemplar foi transferido para o tanque principal com capacidade total de $34000 \mathrm{I}$.

\section{DISCUSSÃO E CONCLUSÕES}

Nascimento e reprodução em cativeiro são eventos distintos que devem ser descritos de forma clara e concisa; na reprodução são observados a cópula, o desenvolvimento (de ovíparo a vivíparo aplacentário) e o nascimento. O nascimento das raias em cativeiro, possivelmente, foi estimulado pelo stress durante a captura e transporte da fêmea (GILBERT 1981). Nos trabalhos de reprodução com elasmobrânquios em cativeiro devem ser observados os vários modos de desenvolvimento reprodutivo encontrados em tubarões e raias. As grandes diferenças encontradas, desde a oviparidade até a viviparidade aplacentária, dificultam a adoção de técnicas gerais (DEMSKı 1990). Por esta razão deve-se realizar procedimentos padrões para cada tipo de modo reprodutivo.

De acordo com UchidA et al. (1990), são poucas as informações sobre reprodução de el asmobrânquios em cativeiro, princi pal mente quando relacionados à sobrevivência e desenvolvimento de filhotes. Os primeiros trabal hos bem sucedidos com reprodução de raias; UCHIDA et al. (1990) com Aetobatus narinari (Euphrasen, 1790), Throson et al. (1983) com Potamotrygon motoro (Müller \& Henle, 1841) e LueR \& GILBERT (1985) com Raja eglanteria Bosc, 1800; demonstraram grande otimismo com a possibilidade do desenvolvimento de novas técnicas. No Brasil existem dois registros sobre reprodução e nascimento de elasmobrânquios em cativeiro (Gonzalez 1996-2002).

Para garantir o sucesso na reprodução, no nascimento e no desenvolvimento de elasmobrânquios em cativeiro, o esforço de captura, transporte e quarentena devem ser o menos estressante possível e a alimentação e a qualidade do cativeiro estejam similares à do meio em que o animal vive (UCHIDA et al. 1990, SMITH 1992, GonZALEZ 1999). O período de quarentena é um dos mais importantes em todo o procedimento, possibilitando o tratamento de infecções e possíveis danos causados aos animais durante a captura e transporte (SMITH 1992).

Infelizmente o aumento na demanda por elasmobrânquios para manutenção em cativeiro vem gerando inúmeros problemas para as populações costeiras do nosso litoral, sendo justamente a preservação destes peixes o objetivo de maior importância dos estudos com reprodução em cativeiro (DEM SKI 1990).

\section{AGRADECIMENTOS}

Ao biólogo Carlo Magenta da Cunha pela leitura e sugestões; ao Dr. Wagner Intelizano e aos biólogos Renan Ruas Reis, Carlo Magenta da Cunha e Luiz Gustavo Aleagi pelo auxílio empregado nos cativeiros durante este trabal ho.

\section{REFERÊNCIAS BIBLIOGRÁ FICAS}

BATISTA, V.S. 1987. Desenvolvimento sexual de Zapteryx brevirostris (Müller \& Henle, 1841), no litoral do Rio de Janeiro, Brasil. Revista Brasileira de Biologia, Rio de Janeiro, 47 (3): 301-307.

DeMSKI, L.S. 1990. Elasmobranch reproductive biology: implications for captive breeding. J ournal of Aquariculture and Aquatic Sciences, Kansas City, 5 (4): 84-95.

Figueiredo, J.L. 1977. Manual de peixes marinhos do sudeste do Brasil. I. Introdução, cações, raias e quimeras. São Paulo, Museu de Zoologia da Universidade de São Paulo, 104p.

GILBERT, P.W. 1981. Patterns of shark reproduction. Oceanus, New Bedford, 24 (4): 30-39.

GonZALEZ, M.M.B. 1996-2002. South American captive elasmobranch census. In: E. FIRCHAU (Ed). International Captive Elasmobranch Census. Virginia, American Elasmobranch Society Technical Report.

. 1999. Hifalomicose em Mustelus schmitti (Springer) (Chondrichthyes, Triakidae). Revista Brasileira de Zoologia, Curitiba, 16 (2): 293-298.

Gruber, S.H. \& R.S. KeYES. 1981. Keeping sharks for research, p. 373-402. In: A.D. Hawkins (Ed.). Aquarium systems. New York, Academic Press, 452p.

HamletT, W.C. \& T.J. Коов. 1999. Female reproductive system, p. 398-443. In: W.C. Ham LETT (Ed.). Sharks, skates and rays. The biology of elasmobranchsfishes. Maryland, TheJohn Hopkins University Press, 515p.

INTERNATIONAL ZOo YeARBOOKS. 1963-2003. Zoological Society of London. London, vols 3-38.

LueR, C.A. \& P.W. GILBeRT. 1985. Mating behavior, egg deposition, incubation period, and hatching in the clearnose skate, Raja eglanteria. Environmental Biology of Fishes, London, 13: 161-171.

Menni, R.C. \& M.F.W. Stehmann. 2000. Distribution, environment and biology of batoid fishes off Argentina, Uruguay and Brazil. A review. Revista Museo Argentino de Ciencias Naturales, Buenos Aires, 2 (1): 69-109.

MuRRU, F.L. 1990. The care and maintenance of elasmobranchs in controlled environments, p. 203-209. In: H. R. Pratt, S.H. Gruber \& T. Taniuchi (Eds.). Elasmobranchs as Living Resources: Advancesin the Biology, Ecology, Systematics, and the Status of the Fisheries. Seatle, NOAA technical Report NMFS 90, 518p.

SMITH, M.F.L. 1992. Capture and transportation of elasmobranchs, with emphasis on the grey nurse shark (Carcharias taurus). Australian Journal of Marine and Freshwater Research, Collingwood, 43: 325-343.

Thorson, T.B.; J.K. Langhammer \& M.I. Oetinger. 1983. Reproduction and development of South American freshwater stingrays, Potamotrygon circularis and P. motoro. Environmental Biology of Fishes, London, 9: 3-24. 
UCHIDA, S.; M. ToDA \& Y. KAMEI. 1990. Reproduction of elasmobranchs in captivity, p. 211-237. In: H. R. PratT; S.H. Gruber $\&$ T. TANIUCHI (Eds). Elasmobranchs as Living Resources:
Advances in the Biology, Ecology, Systematics, and the Status of the Fisheries. Seatle, NOAA Technical Report NMFS 90, 518p.

Recebido em 02.III.2004; aceito em 28.IX.2004.

Revista Brasileira de Zoologia 21(4): 785-788, dezembro 2004 